\title{
OCIO SERIO E IDENTIDAD SOCIAL. ELEMENTOS PARA UN ANÁLISIS SOCIOLÓGICO DE LA PARTICIPACIÓN POPULAR EN PRUEBAS DEPORTIVAS DE FONDO
}

\author{
Manuel Ángel Santana Turégano* \\ masantur@ull.es \\ Universidad de La Laguna
}

\section{RESUMEN}

El objetivo de este trabajo es desarrollar una perspectiva para el análisis del crecimiento en la participación popular en pruebas deportivas de fondo. Para comprender este fenómeno se parte de la perspectiva del ocio serio, que combinada con la teoría de la identidad social permite plantear como hipótesis que el crecimiento de estas pruebas se explica porque permite, a quienes participan en ellas, construir su identidad social en positivo, mediante dos mecanismos. Por un lado, permite alcanzar una posición elevada en al menos una jerarquía social, la propia de las subculturas asociadas a determinadas prácticas deportivas. Por otro lado, en la medida en que el ethos de estas prácticas resulta coherente con el de las sociedades contemporáneas, permite mejorar, en un sentido más genérico, la propia identidad social.

Palabras Clave: ocio serio, deportes de fondo, maratón, triatlón, ciclismo, identidad social.

\author{
SERIOUS LEISURE AND SOCIAL IDENTITY. ELEMENTS \\ FOR A SOCIOLOGICAL ANALYSIS OF POPULAR \\ PARTICIPATION IN ENDURANCE SPORTS
}

\section{Abstract}

The aim of this paper is to develop an approach to analyze the growth in the participation of amateur athletes in endurance sports events. Following the serious leisure perspective, combined with social identity theory, an approach is proposed based in the hypothesis that the growth of these events can be explained because taking part in them allow participants to build a positive social identity through two mechanisms. On the one hand, it allow participants to gain a high ranking in at least one social hierarchy, that of the subculture of certain sports. On the other hand, for as long as the ethos of endurance sports is consistent with that of contemporary societies, it also allows, in a more general sense, to enhance one's own social identity.

KEYWORDs: serious leisure, endurance sports, marathon, triathlon, cycling, social identity.

DOI: https://doi.org/10.25145/j.atlantid.2019.10.08 


\section{INTRODUCCIÓN}

En los últimos años se ha dado un importantísimo crecimiento en la participación popular en maratones, carreras de trail-running, triatlones, pruebas ciclistas y en general en todo tipo de competiciones populares de deportes de resistencia ${ }^{1}$. A título de ejemplo, baste pensar que para 2018 podría estimarse que unas 65000 personas terminaron alguno de los 32 maratones que se celebraron en España (cerca del 0,1\% de la población, Novatos del Running, 2018), y que en los Estados Unidos podría estimarse que en torno a medio millón de personas terminan un maratón al año (el 0,5\% de la población, Runners World, 2017); que también en torno a medio millón de personas, pero en este caso en todo el mundo, terminan cada año un triatlón de distancia ironman (Zahumensky, 2018), y así podríamos seguir con las carreras de montaña o las pruebas ciclistas, tanto en ruta como en bicicleta de montaña. Obviamente, la creciente participación popular en pruebas deportivas de fondo se enmarca dentro del incremento general de la práctica deportiva: mientras que en 1975 sólo el 22\% de la población española practicaba deporte con regularidad, para 2015 puede estimarse que casi la mitad de la población practica algún deporte con regularidad al menos semanal (García Ferrando, 2006; Consejo Superior de Deportes, 2015), y los porcentajes son similares tanto para Norteamérica (Kolokakis et al., 2012) como para el conjunto de la Unión Europea (Comisión Europea, 2015). Y dentro del incremento general de la práctica deportiva, los deportes de fondo son aquellos cuya práctica más se ha incrementado: de acuerdo a los datos del Consejo Superior de Deportes (2015) puede estimarse que entre las actividades deportivas más practicadas por los españoles se encuentran aquellas que suelen considerarse deportes de fondo, como pueden ser el ciclismo $(38,7 \%$ de la población que practica deporte), el senderismo-montañismo (31,9\% de la población que practica deporte) y la carrera a pie $(30,4 \%)$ y la natación $(38,5 \%)^{2}$.

* Universidad de La Laguna, Facultad de Economía, Empresa y Turismo, Departamento de Sociología y Antropología Social, Apartado 456, San Cristóbal de La Laguna-Tenerife.

${ }^{1}$ Los deportes de resistencia podrían definirse como aquellos en que se ejerce una actividad física submáxima durante períodos de tiempo prolongados (varias horas). Desde un punto de vista médico se diferencia en ocasiones entre deportes que requieren un esfuerzo de entre media hora y dos horas y aquellos que implican un esfuerzo superior a las dos horas, entre los cuales Engelhardt et al. (1998), por ejemplo, incluyen maratones, ultramaratones y triatlones de larga distancia. En este trabajo consideraremos como deportes de resistencia aquellos que implican un esfuerzo superior a la hora, hora y media de duración, lo que incluiría, en atletismo carreras a partir de la distancia de medio maratón, en triatlón competiciones a partir de la distancia olímpica y en carreras de trail, en ciclismo (carretera o montańa) y otro tipo de competiciones todos aquellos eventos que llevan al participante medio a superar la hora y media de esfuerzo para completarlos.

${ }^{2}$ En realidad, existen modalidades de ciclismo, como el ciclismo en pista, que no pueden considerarse un deporte de fondo, y lo mismo sucede con el atletismo: los 100 metros lisos no son una prueba de fondo, aunque en el imaginario colectivo (y en la práctica popular) el atletismo se asocia más a carreras como el medio maratón o el maratón, que son claramente deportes de fondo. En el caso de la natación es justamente al revés: si bien existen pruebas que son claramente de fondo, quizá las más conocidas son las que reclaman otro tipo de cualidades. 
Los motivos para el incremento general de la práctica deportiva son variados, y exceden los objetivos de este trabajo (mayor disponibilidad de tiempo de ocio y mayor sedentarismo en el trabajo). Dentro de este incremento, que sean justamente los deportes de fondo aquellos que hayan experimentado un mayor crecimiento en su práctica se puede comprender también por distintos motivos, tanto médicos ${ }^{3}$ como sociales y económicos ${ }^{4}$. Sin embargo, el incremento de la participación popular en pruebas de fondo no puede explicarse por estos motivos: no sólo no resulta barato, sino que cuesta dinero (bastante). Y, a menudo, la práctica de este tipo de actividades implica problemas de salud que llevan a millones de personas a la consulta de médicos y fisioterapeutas. Cada año varios millones de personas de todo el mundo dedican grandes cantidades de tiempo y dinero para entrenar y participar en pruebas deportivas de fondo, y no reciben por ello ninguna remuneración o recompensa, más allá de la satisfacción de superar un reto. Se trata además de prácticas que tan sólo pueden considerarse como inmediata e intrínsecamente gratificantes en un sentido un tanto sui generis (en general, se dice que "se disfruta sufriendo»). ¿Qué lleva a tanta gente a hacer lo que aparentemente parece una "locura»? En este artículo trataremos de dar respuesta a esta pregunta. Para ello se plantea un marco teórico para el análisis sociológico de la participación popular en pruebas deportivas de fondo desde las teorías de la identidad social (Stets y Burke, 2000) y la perspectiva del ocio serio (Serious Leisure Perspective, SLP, véase, por ejemplo, Stebbins, 2006), si bien en apartados importantes nos alejaremos de la misma.

En las sociedades contemporáneas, que algunos autores han denominado «sociedades del ocio», un número cada vez más importante de personas disponen de tiempo y recursos importantes para dedicar a las actividades de ocio, lo que otorga más importancia a las actividades relacionadas con lo que se ha dado en llamar «ocio serio" (Stebbins, 2006; Elkington y Stebbins, 2014), e implica una importante ampliación del abanico de actividades de ocio posibles. En la estructura social propia de las sociedades avanzadas el ocio es un ámbito que permite construir una identidad social positivamente valorada, a diferencia de lo que sucedía en épocas anteriores, en las que el ocio era fundamentalmente un ámbito en que demostrar la posición que se ocupaba en el sistema productivo. Surge por lo tanto todo un conjunto de actividades que, aunque son de ocio, pues no se percibe recompensación monetaria por practicarlas, se practican seriamente (ocio serio), ya que permiten a los individuos construirse una identidad social (soy corredor, soy triatleta, soy músico). En función de la relevancia que puedan llegar a tener (identity salience), algunas de estas actividades pueden llegar a convertirse en elementos claves para

${ }^{3}$ En general, especialmente a partir de una edad, las recomendaciones médicas son las de practicar una «actividad física modera/aeróbica», y los deportes de fondo cumplen con estos requisitos.

${ }^{4}$ Por más que sea tópico no deja de ser cierto lo que se suele decir de que «necesitas juntar a 22 amigos/as para jugar un partido de fútbol, mientras que no necesitas a nadie para ir a correr; necesitas tener un campo de golf y un equipo caro para jugar al golf, mientras que con unas zapatillas y una calle medianamente tranquila puedes empezar a correr». 
la identidad social de muchos individuos, y esto es lo que explicaría que se invierta tanto tiempo y recursos en ellas.

Los fenómenos de construcción de la identidad a través del ocio no son nuevos, lo novedoso son los ámbitos y mecanismos a través de los cuales se realizan. Así, por ejemplo, durante la segunda mitad del siglo xx en los países comunistas, en los cuales oficialmente no existían clases, y por lo tanto la posición que cada quien ocupaba en la estructura social no era tan relevante, se dio un extraordinario desarrollo de actividades de "ocio serio" ${ }^{5}$, fundamentalmente en el deporte, la música y otro tipo de artes (ballet, por ejemplo $\left.{ }^{6}\right)$. La hipótesis de trabajo de este artículo será que existe una homología estructural entre las prácticas de ocio de una sociedad y el ethos que impera en la misma, y aquellas actividades de ocio que permitan desarrollar una identidad social más acorde con los valores dominantes serán aquellas que se persigan de manera más masiva ${ }^{7}$. Por otro lado, en la medida en que una determinada actividad de ocio encarne valores relativamente distintos (subcultura) o directamente opuestos a los dominantes (contracultura), la práctica de la misma será una mayor fuente de identidad para quienes se involucren en ella, con lo que nos podemos encontrar con actividades cuya práctica es menos masiva pero más intensa. En definitiva, el auge actual de determinadas actividades de ocio, como la participación en maratones, triatlones, carreras de trail-running y pruebas ciclo deportivas, se explicaría porque, de acuerdo al ethos contemporáneo, la participación en las mismas permite construir una identidad social que es valorada positivamente.

Para desarrollar este argumento, tras hacer una revisión de la literatura existente se expondrán los conceptos de ocio y ocio serio, y se analizará cómo ha cambiado el papel que el ocio desempeña en la identidad social en el último medio siglo. A continuación, se tratarán las teorías que permiten comprender cómo se construye la identidad social, comparando las aportaciones psicológicas y sociológicas al respecto, así como los cambios que en los últimos tiempos se han dado en el mundo del ocio. Por último, se expondrán algunos elementos del cambio acerca de las valoraciones sociales de la identidad en los últimos años. De los resultados de este enfoque se deducen importantes implicaciones, tanto teóricas, pues cada vez parece más necesario el análisis del ocio para comprender las sociedades contemporáneas, como prácticas, que pueden ayudar en la planificación y gestión, tanto

5 Aunque el concepto de ocio serio fuera desarrollado durante la guerra fría del otro lado del telón de acero. Sobre el ocio en los países comunistas puede verse Roberts y Fagan, 1999 y Roberts et al., 2005.

${ }^{6}$ Desde el punto del individuo la implicación en estas actividades constituía, especialmente para quienes era exitosos en las mismas, una parte de la identidad social más importante que la que se derivaba del trabajo. Desde el punto de vista de la sociedad, estas actividades eran útiles, sobre todo, en la medida en que permitían el enaltecimiento del grupo (de los valores socialistas), y por ello la capacidad de derrotar al adversario era una cuestión clave.

${ }^{7}$ A modo de botón de muestra: en una sociedad individualista experimentan un auge los deportes individuales (maratones, triatlones, ciclismo, pádel, tenis); si bien es cierto que se siguen considerando un deporte de equipo. 
pública como privada, del importante sector que se ha generado en torno a las pruebas deportivas populares.

\section{OCIO Y OCIO SERIO EN LAS SOCIEDADES CONTEMPORÁNEAS}

Desde principios del siglo xxi existe una abundante literatura que analiza la participación popular en maratones, pruebas ciclistas o triatlones, entre otros eventos deportivos. Así, a modo de ejemplo, y sin pretensiones de exhaustividad, en el ámbito del atletismo de larga distancia Shipway y Jones (2007) realizaron una investigación etnográfica para comprender las motivaciones de los participantes en un evento de turismo deportivo celebrado en Chipre. Usaron la misma metodología de investigación para comprender la perspectiva subjetiva de los participantes en uno de los mayores maratones del mundo (Shipway y Jones, 2008), y en una línea similar Shipway y Holloway (2010) analizaron la relación entre la subcultura del running y la adopción de un estilo de vida saludable. Fairer-Wessels (2013) usó también técnicas cualitativas para comprender las motivaciones de los participantes en el Comrades Marathon, celebrado cada año en Sudáfrica. Desde una perspectiva distinta, la de la Behavioural Economics, Allen et al. (2016) analizaron el comportamiento (deportivo) de los participantes en los maratones a partir del concepto de «preferencias dependientes de las referencias». Y desde un enfoque también distinto, Papanikos (2015) ha analizado el impacto económico de un maratón en tanto que evento de turismo deportivo. En español, Ruiz Juan y Zarauz-Sancho (2014) han analizado la motivación en los corredores de maratón españoles. Siguiendo con la perspectiva psicológica, Jaenes et al. (2009) han analizado los tipos de personalidad que se asocian con la participación en eventos como el maratón, mientras que Fernández Macías et al. (2015) han analizado el conocido como «estado de flow» en maratonianos/as. En el mundo del ciclismo Shipway et al. (2016) han analizado la participación en una prueba que combina el ciclismo activo (de carretera) con el de espectador, desde la perspectiva del ocio serio. Siguiendo en esta línea, y desarrollando el concepto de «deporte serio» (serious sport), Getz y MacConnell (2011) han analizado el desarrollo de una "carrera» dentro del ciclismo de montańa, mientras que Fairley et al. (2011), aplicando un marco conceptual más cercano al turismo, han utilizado el concepto de "nostalgia» para comprender la implicación y participación en determinados viajes/eventos. Por último, aunque es quizá menos abundante, existe también literatura que analiza la participación en el triatlón. Sleivert y Rowlands (1996), desde una perspectiva cercana a la medicina deportiva, han analizado los factores psicológicos que se asocian con el éxito en triatlón, mientras que desde una perspectiva sociológica cuantitativa García García (2016) ha analizado el triatlón de larga distancia como una subcultura. En definitiva, de la revisión de la literatura existente se deduce que el marco teórico de la mayoría de los trabajos académicos sobre los deportes de fondo se nutre fundamentalmente de la perspectiva del ocio serio (Serious Leisure Perspective, SLP), por lo que pasamos a continuación a explicar los conceptos fundamentales de la misma. Desde esta perspec- 
tiva, el ocio, es decir, aquellas «actividades que las personas eligen realizar durante su tiempo libre y que les dan algún tipo satisfacción, sentimiento de logro o ambas", abarca en realidad tres tipos de fenómenos relativamente diferenciados: ocio informal, ocio serio y ocio basado en proyectos.

El ocio informal es quizá lo que de manera más intuitiva se asocia con el ocio: actividades por las que tienes que pagar, y por las que difícilmente se puede concebir cómo podría ser de otra manera (que te pagaran por hacerlas). Es lo que a menudo se asocia con el «entretenimiento» (entertainment): leer una novela, ir al cine, al teatro, a un espectáculo deportivo o ver en casa deporte, cine o una serie de televisión; salir a cenar, a beber algo, o quedarse en casa y mantener relaciones sexuales. Consiste en la realización de actividades inmediata e intrínsecamente gratificantes, placenteras y de corta duración, que no requieren de una capacitación especial para disfrutarlas y que tienen un carácter hedonista. Incluiría actividades como el juego, la relajación, el entretenimiento pasivo o activo o la estimulación sensorial ${ }^{8}$. Durante buena parte de la Revolución Industrial, para la mayoría de la población el tiempo dedicado a la actividad laboral y a la atención de las obligaciones domésticas y familiares ocupaba la mayor parte del día. Para las clases trabajadoras las jornadas laborales de ocho horas diarias y cinco días en semana son históricamente recientes (y eso sin contar el tiempo de trabajo doméstico), y en cualquier caso el dinero disponible para el ocio era muy limitado, por lo que la mayoría de actividades de ocio, para buena parte de la población, entraban en esta categoría, pues el poco tiempo libre disponible tenía un carácter más bien de «restauración» de los esfuerzos y de reparación/descanso para la dura vida cotidiana, por lo que a la mayoría de personas les quedaría poco tiempo y energías disponibles para otras actividades. En la segunda mitad del siglo xx, para buena parte de las clases trabajadoras, este ocio, que a menudo se ha tachado de manera un tanto peyorativa de "pasivo» o «no creativo", consistía en ver la televisión, acudir al bar o pub local a tomar algo, seguir prensa o radio sensacionalistas y, en los períodos vacacionales, acudir a lo que a menudo se ha denominado «la periferia del placer» a desarrollar actividades mayormente pasivas como los baños de sol'.

Por contraposición a este ocio informal (casual leisure), el ocio serio, tal y como es definido de manera más o menos canónica desde la perspectiva del ocio serio (SLP), sería la práctica sistemática de una actividad no remunerada, que se practica en tanto que aficionado (amateur), voluntario o como un hobby, y que

${ }^{8}$ Los conceptos fundamentales pueden encontrarse en cualquiera de las referencias citadas. Dado que la perspectiva tiene incluso una página web: https://www.seriousleisure.net, a continuación seguiremos lo que en esta se explica para nuestra exposición, haciendo una traducción y adaptación de la misma.

9 Una anécdota: en las zonas turísticas, en los últimos años, se han generalizado unas camisetas souvenirs rotuladas «Spanish Triathlon» (o Canary Islands, Mallorca, o lo que sea): «Eating, drinking, fucking». En lo que puede considerarse una especie de burla de las clases populares hacia la altivez con que son vistas desde las clases altas, se regodean en el carácter pasivo y simple de las actividades de ocio que practican, mofándose también de las extenuantes actividades que practican en su tiempo libre otros, ejemplificados en los triatletas (nadar, hacer bicicleta, correr). 
resulta importante, interesante y significativa, en cuanto que ayuda a encontrar una dirección y/o sentido a la vida, para las personas que la practican. El concepto surge, a mediados de la década de 1970, a partir de los trabajos que realizó Stebbins sobre músicos, cómicos, actores y científicos amateurs (véase Stebbins, 1976), que dieron lugar al surgimiento del concepto de ocio serio (véase Stebbins, 1977, 1982). Cuando surge la perspectiva del ocio serio, en Estados Unidos en torno a la década de 1980, permite dar cuenta de un fenómeno aparentemente contradictorio y hasta entonces incomprensible: que la gente se esforzara tanto en el ocio, pues ocio y serio parecían prácticamente opuestos. En este sentido, resulta de ayuda para comprender fenómenos como la participación popular en maratones, pruebas ciclistas o triatlones, que requieren de mucha seriedad, pues sólo para conseguir llegar a la meta en uno de estos eventos hay que dedicar muchas horas a entrenamientos, y dinero en material para entrenar, entre otras cuestiones. En la actualidad, la perspectiva del ocio serio se ha convertido en la teoría dominante en la academia, y adherirse a la misma facilita en gran medida la publicación en los círculos que hoy en día permiten el avance en el entorno académico. Sin embargo, creemos que es necesario, antes de continuar, señalar algunas de sus limitaciones, y en qué medida nos distanciaremos de ella en este trabajo.

En primer lugar, un aspecto en el que nos apartaremos en parte de la perspectiva de ocio serio, tal y como esta suele aplicarse, es que la diferencia entre practicar una actividad de ocio, de ocio serio y trabajar es en último término una cuestión de quién paga qué. El ocio informal (casual leisure) implica casi siempre la participación de otras personas en cuanto que trabajadores: por la misma actividad unos pagan y otros cobran ${ }^{10}$. Por lo tanto, es muy importante remarcar que, si lo que diferencia una actividad de ocio serio de una actividad profesional es que las actividades de ocio serio no son remuneradas, la diferencia entre unas y otras es, y así ha sido históricamente, más una cuestión de grados que una diferenciación dicotómica. Aunque en los trabajos empíricos que se han realizado esta diferenciación quizá no ha tenido la atención que merecía, en la clasificación del ocio (leisure) cabe diferenciar entre "ocio informal» $\mathrm{y}$ "actividades serias" (serious pursuits), y dentro de estas se incluirían tanto el ocio serio (serious leisure) como lo que se podría traducir como «trabajo apasionado» (devotee work ${ }^{11}$ ). En cuanto a las actividades que aquí nos ocupan, los deportes de fondo son una profesión para unas pocas personas, que viven, y muy bien, de ello ${ }^{12}$. En el extremo contrario, a muchas personas les cuesta mucho

10 Si veo una película, voy a un concierto, asisto a un espectáculo deportivo o voy a cenar, mi ocio implica que otras personas me procuran lo que de gratificante y placentero tiene esa actividad (actores, músicos, deportistas), y que en la mayoría de los casos realizan lo que para ellas es un trabajo (viven, como se dice en inglés, del show business).

${ }^{11}$ Esta diferenciación puede encontrarse en https://www.seriousleisure.net/slp-diagrams. html.

12 Los ciclistas profesionales tienen contratos millonarios, a la altura, en algunos casos, de otras estrellas mediáticas; y los corredores y triatletas profesionales, sin llegar a estos niveles, viven también muy bien de lo que para otras personas es tan sólo una afición (ocio serio). 
dinero la misma actividad que a otras da dinero, y no sólo porque compren material (bicicletas, zapatillas) o servicios (cuotas de piscinas, gimnasios, entrenadores), sino porque incluso han de pagar para participar en eventos como corredores, triatletas o ciclistas ${ }^{13}$. Pero entre ambos extremos, el profesional, que vive bien de una actividad, y el completamente amateur, que paga todos y cada uno de los costes asociados al desarrollo de una actividad, hay un amplio abanico de situaciones intermedias. En una gradación desde más amateur (ocio serio) a menos (negocio), existen corredores, ciclistas y triatletas que son lo suficientemente "buenos/as» como para que los inviten a determinadas competiciones; otros tan buenos que además de invitarlos a algunos eventos alguna empresa del sector los patrocina dándoles material y/o servicios para practicar la actividad de que se trate (bicicletas, zapatillas, entrenadores), con lo que no tienen que ponérselo todo de su bolsillo. El último paso antes del profesionalismo sería el de aquellas personas que son lo suficientemente buenas en una actividad como para recibir alguna remuneración por ello, pero no lo bastante como para vivir de la misma ${ }^{14}$. Y algo similar puede decirse de las actividades que sirvieron a Stebbins originariamente para desarrollar el concepto de ocio serio: así, por ejemplo, el electricista que toca en un trío y que un par de fines de semana al mes toca en bodas y otros eventos y se saca con ello un dinero extra; el mecánico que da clases de salsa en una academia y entre las dos actividades se saca un buen sueldo; el historiador, arqueólogo o astrónomo «aficionado» que es profesor en un instituto, pero al que ocasionalmente pagan por dar conferencias.

En segundo lugar, otra cuestión en que nos apartaremos de otros estudios realizados desde la perspectiva del ocio serio es que es necesario también matizar que la idea de que una actividad de ocio serio es aquella que de alguna manera otorga una identidad social y ayuda a la persona a encontrar un sentido y dirección a su vida, por contraposición a las actividades de «ocio informal», implica también todo un abanico de posiciones y no una diferenciación dicotómica, y esto se ha acentuado en los últimos ańos. Hasta cierto punto, podría decirse que en la actualidad actividades de ocio claramente pasivas, como ser seguidor de una teleserie (Star Trek, Big Bang Theory), de un determinado género (manga, cómic) o amante de un determinado tipo de bebidas (cervezas artesanas), para algunas personas se han convertido en elementos importantes de la identidad social: de la misma manera en que hay quien se define como «soy corredor» (o ciclista) hay quien se define como "soy Trekkie» (o foddie, o winelover ${ }^{15}$ ). Por último, hay que reseñar que además de las categorías

13 A título de ejemplo, para 2019 se puede estimar que participar en triatlón distancia ironman cuesta más de 200 euros; un maratón importante se acerca a los 60-70 euros, cifra similar para una prueba ciclodeportiva.

${ }^{14}$ Por último, en aquellos casos en que se pueda vivir, más o menos bien, de lo que para otras personas es una afición, estamos ya en el ámbito del profesionalismo, por mal remunerado que pueda estar, y no ya en el del ocio.

${ }_{15}$ En principio, al menos desde la mentalidad propia de la modernidad avanzada, tendería a pensarse que hay actividades que para unas personas son ocio, pero que también pueden ser negocio para otras personas, y que por lo tanto serían más susceptibles de convertirse en formas de ocio serio 
de ocio informal (casual leisure) y ocio serio (serious leisure), a partir de 2005 (véase Stebbins, 2005) se ha desarrollado un concepto que da cuenta de un tercer tipo de ocio: el Project-based leisure (ocio basado en proyectos). El ocio basado en proyectos sería la realización de tareas moderadamente complicadas, que requieren planificación, esfuerzo y en ocasiones habilidades y/o conocimientos, pero que se realizan de forma ocasional o infrecuente, por lo que podría decirse que se encuentra, de alguna manera, entre los dos tipos de ocio anteriormente mencionados. En cualquier caso, es importante hacer notar que, aunque unas actividades tienen mayor potencialidad que otras para convertirse en ocio serio, que una actividad pueda considerarse ocio informal, ocio serio u ocio basado en proyectos depende en gran medida del sentido subjetivo de quien la realiza.

Desde la perspectiva del ocio serio se ha llegado al consenso de que las actividades de ocio serio tienen seis características distintivas (Romero, Iraurgi y Madariaga. 2017; Elkington y Stebins 2014): 1) En primer lugar, que es necesario perseverar para llevar a cabo la actividad, 2) en segundo lugar, que existe una especie de trayectoria de progresión dentro de la actividad (career), que es reconocida por quienes la practican, 3) en tercer lugar, que la progresión en esta trayectoria sólo se logra mediante el esfuerzo en conseguir habilidades y/o conocimientos, 4) en cuarto lugar, que la práctica de la actividad permite obtener una serie de beneficios duraderos, como pueden ser la autoexpresión o el sentimiento de logro, 5) en quinto lugar, que es posible identificar, hasta cierto punto, un ethos (forma de vida y sistema de valores) asociado a una actividad, 6) en sexto y último lugar, una actividad puede convertirse en ocio serio si permite desarrollar una identidad personal y social ligada a la actividad de ocio serio que es vista como socialmente atractiva al menos en algunos grupos sociales. Si combinamos una determinada actividad con el acercamiento subjetivo que una persona realice a la misma, podría decirse que, en la medida en que se cumplan más o menos cada una de las seis características que acabamos de mencionar, nos acercaríamos al «tipo ideal» de ocio serio. Para intentar recoger la variabilidad de estos fenómenos se ha desarrollado una herramienta de medida, el Serious Leisure Inventory Measure (Gould et al., 2008), que intenta reflejar cada una de estas seis características. En una reformulación más reciente (Gould et al., 2011) esta herramienta se ha rediseñado para poderse utilizar como medida de en qué grado una persona está comprometida en una determinada actividad de ocio, y se ha aplicado al deporte del surf (Barbieri y Sotomayor, 2013; Sotomayor y Barbieri, 2016), jugadores de béisbol y voleibol (Liu et al., 2013) y a una muestra variada de deportistas federados (Romero, Iraurgi y Madariaga, 2017).

(los ejemplos clásicos: música, deportes, teatro/cine), mientras que otras actividades, que constituyen el trabajo de muchas personas, difícilmente pueden convertirse en ocio para otras. En cualquier caso, el surgimiento de actividades como el trainspotting o el tuneado de vehículos, que podrían entenderse, al menos en parte, como una reinvención en el mundo del ocio de actividades originadas en el negocio, nos deberían hacer cautos al respecto. Por último, fenómenos como el surgimiento de ligas y profesionales de los e-sports nos debería hacer cautos también a la hora de pensar que determinadas formas de ocio "pasivo» no puedan convertirse también en ocio serio o incluso en negocio. 
En cualquier caso es importante recordar que la diferenciación entre ocio informal (casual leisure), ocio basado en proyectos (project based leisure), ocio serio (serious leisure) y trabajo apasionado (devotee work) es una cuestión de gradación, y que además es posible encontrar cambios y evoluciones, tanto a lo largo de la vida de una persona y en implicación con una actividad como la evolución de las propias actividades ${ }^{16}$. Además, es importante tener en cuenta que en los modos de organización laboral postfordistas se intenta a menudo lograr la implicación de los trabajadores/as en sus tareas, que vean su trabajo como algo más que un mero trabajo, con lo que podría decirse que el trabajo apasionado (y apasionante, «devotee work»), que sería el siguiente grado de compromiso con una actividad tras el del «ocio serio», se acaba convirtiendo casi en el trabajo ideal para el ethos de las sociedades contemporáneas. Desde la perspectiva del ocio serio se subraya la aspiración de muchas personas de encontrar sentido y satisfacción en lo que hacen en su tiempo libre como una motivación para el desarrollo de las actividades de ocio serio. Pero creemos que es importante tener en cuenta que a menudo lo que sucede es que la aspiración de encontrar sentido y satisfacción en lo que se hace está en la base de la implicación de muchas personas en muchas actividades, que en el sentido subjetivo que les dan sus protagonistas, no son tan sólo de ocio, sino que tienen algún tipo de aspiración de negocio, que es a menudo superior en las actividades emergentes y que por lo tanto están en crecimiento. Entre los varios miles de participantes de las pruebas más importantes de triatlón, maratón, trail-running o ciclismo popular, no llegan al $0,1 \%$ quienes pueden aspirar a convertirse en deportistas profesionales, pero el enorme crecimiento de estas actividades puede alimentar las expectativas de un porcentaje de participantes mucho mayor de convertirse en cierta manera en "profesionales» de lo que para otras personas es tan sólo una actividad de ocio (de ocio serio) en un sentido atípico. Mediante la implicación en la actividad y la consecución de determinadas prestaciones puede acumularse un capital simbólico y relacional que puede convertirse en un negocio lucrativo en el emergente sector dedicado a estas actividades ${ }^{17}$. En la línea de lo que señala Hernández (2017), en una sociedad

16 Así, por ejemplo, no es extraño iniciarse en una actividad por motivos de salud, con lo que apenas cabe considerar eso una actividad de ocio; posteriormente se encuentra gusto en la misma, con lo que puede llegar a convertirse en una actividad de ocio serio; y, en algunos casos, puede llegar a convertirse en una profesión (trabajo apasionado, devotee work). Cuando surge una nueva actividad no existen profesionales de la misma, pero a medida que esta se va consolidando se convierte en una alternativa laboral para muchas personas: así, por ejemplo, en la década de 1980 no cabía hablar propiamente de profesionales del triatlón, mientras que en la actualidad hay un amplio número de personas (entrenadores, organizadores, vendedores de material) que se ganan la vida en ese ámbito. En la actualidad es posible que algo similar esté pasando en el mundo del trail-running.

17 Terminar un maratón en dos horas y media no te va a permitir ganarte la vida con el atletismo... como corredor. Pero puedes montar una tienda de zapatillas y material, puedes convertirte en entrenador, o fisioterapeuta; aunque hayas terminado varios triatlones distancia ironman con una buena marca, quizá no te paguen por competir, pero puedes montar una tienda y ganarte la vida con el triatlón; y, pese a que en Italia algunos de los ciclistas que han destacado en las Gran Fondo ciclistas han llegado a profesionales, terminar con buenos tiempos lo que en el mundillo se conoce como 
que pide de los individuos la «excepcionalidad», lo que para unas personas pueden ser prácticas de ocio serio pueden servir a otras para destacar, ser «excepcionales» y progresar en los negocios ${ }^{18}$.

Además de los anteriormente mencionados, desde la perspectiva del ocio serio se han desarrollado investigaciones para medir en qué medida la implicación en actividades de ocio serio se asocian con el mayor o menor desarrollo de determinados rasgos de la personalidad, tanto entre los maratonianos (Hungerberg y Gould, 2015; Romero, Iraurgi y Madariaga, 2017) como en personas que se implican en actividades artísticas (Liu, 2014). Desde una perspectiva no muy distinta, las variables psicológicas se han utilizado para explicar qué motiva a algunas personas a participar en maratones (Francisco-Ruiz y Zarauz-Sancho, 2014). De forma un tanto simplificada podría decirse que esta perspectiva de investigación acaba respondiendo a la cuestión «qué lleva a tanta gente a hacer tantas locuras como correr maratones» en términos de «tienen algo en su constitución psicológica que los lleva a ello». Es habitual en psicología distinguir entre "motivación intrínseca» y «motivación extrínseca». Aplicada esta distinción al tipo de actividades que nos ocupan se podría decir que tienen motivación extrínseca por correr maratones, triatlones o pruebas ciclistas de larga distancia aquellas personas a las que lo que los motiva para realizar estas actividades no es tanto que la práctica en sí les resulte especialmente placentera, sino las consecuencias físicas, grupales y sociales de dicha práctica. Por el contrario, tendrían motivación aquellas personas a las que les guste la práctica deportiva en sí. Desde este punto de vista, y dado que las sensaciones que se experimentan al practicar este tipo de actividades son un tanto particulares, y no siempre resultan placenteras, o al menos no lo que habitualmente se entiende como placenteras, quienes practican deportes de fondo serían personas un tanto distintas, con una cierta tendencia masoquista ${ }^{19}$. En otras ocasiones, la motivación para la práctica de este tipo de actividades se encuentra en lo que en términos psicológicos se conoce como «estado de flow» (al respecto puede verse Fernández Macías et al., 2015) y la generación de hormonas mediante la práctica de estas actividades, que se compara a veces con un chute de drogas sin efectos negativos (el runner's high, o «subidón del corredor», que puede hacerse extensible a otros deportes de fondo). Lo cual, en último término, podría considerarse una motivación casi tan extrín-

«cicloturistas» difícilmente te permitirá convertirte en ciclista profesional, pero si te haces experto en biomecánica, quizá puedas vivir del ciclismo. Como se puede ver en todos estos ejemplos, no basta con ser corredor (o triatleta o ciclista): si no sabes llevar un negocio, no te ganarás la vida con ello.

${ }^{18}$ Así, por ejemplo, si ya eres fisioterapeuta, o tienes un título universitario en Educación Física, una tienda, empresa de eventos u otro tipo de negocio relacionado con estos ámbitos quizá sea mejor «invertir» en acumular capital simbólico logrando determinadas prestaciones, y sobre todo el reconocimiento social que se asocia a las mismas, que en un máster de especialización.

${ }^{19}$ Como se dice a menudo en los círculos de estos deportes, especialmente cuando se pretende remarcar la identidad de grupo frente a otros grupos, «somos de otra pasta (somos tipos/as duros/as que nos gusta sufrir y nos sabemos sacrificar)». 
seca como intrínseca: corres porque a veces, cuando corres, tienes un subidón (con lo cual, las otras veces que corres es un medio).

En cualquier caso, las explicaciones de carácter psicobiológico podrían ayudar a entender por qué unas personas serían más propensas que otras a determinadas actividades. Por la configuración de sus circuitos neuronales serían más sensibles a los estímulos positivos derivados de la actividad, y/o menos sensibles a los estímulos negativos. El estado actual del conocimiento científico en psicología de la personalidad y psicología evolutiva (véase Neetle, 2009) plantea que los cinco grandes rasgos de la personalidad son, en buena medida, genéticos. En la medida en que esos factores llevan a unas u otras prácticas de ocio, ello es lo que explicaría que unas personas tengan la "capacidad de perseverar» que se requiere para participar en deportes de fondo, mientras que otras, que carecen de ella, se dedicarían a otros deportes y/u otras actividades de ocio, quizá más cercanas al ocio informal que al ocio serio. Existe literatura que analiza, por ejemplo, la propensión al neuroticismo entre los corredores de maratón, o si los participantes habituales en pruebas de fondo son más sensibles a experimentar los «estados de conciencia alterada" que se asocian en ocasiones a la realización de esfuerzos físicos extenuantes. Esta literatura ayuda a entender por qué unas personas participan en pruebas deportivas de fondo y otras no, pero no son de gran ayuda a la hora de entender el incremento que se ha dado en la participación en estas pruebas en los últimos tiempos. Como es innegable el hecho de que esta se ha incrementado de manera exponencial en los últimos 40 años, para comprender el auge de la participación popular en las pruebas deportivas de fondo es necesario comprender cómo ha variado la valoración social de las mismas y cómo se construye la identidad social.

\section{LA IDENTIDAD SOCIAL}

En la actualidad el concepto de «identidad» tiende a asociarse con «aquellas cosas que hacen que una persona sea como es", debido a la popularización, un tanto devaluada, de la psicología humanista, por lo que, en cierta medida, tiende a confundirse con el concepto de "personalidad", que ha tenido en los últimos años un extraordinario desarrollo en el campo de la "psicología de la personalidad». Sin embargo, desde el punto de vista sociológico, la identidad se asociaría más bien con «el conjunto de grupos a los que pertenece un individuo que lo definen como tal». En cualquier caso, aun desde las teorías psicológicas la pertenencia de una persona a un grupo resulta clave para la definición de su autoconcepto y de su identidad. Constituyendo un enlace entre lo social y lo psicológico, la teoría de la identidad social plantea que el comportamiento social de una persona se sitúa entre dos polos de un continuo (véase, por ejemplo, Massenzana, 2017). Por un lado, en lo que se considera comportamiento interpersonal, el comportamiento vendría determinado por características personales idiosincráticas (que podrían asociarse a los cinco grandes rasgos de la personalidad vistos anteriormente) y por las relaciones personales con otros individuos y el comportamiento intergrupal, que vendría determinado por la pertenencia a determinados grupos sociales, y que en determinados ámbitos 
predominarían más unos u otros. De acuerdo a la teoría de la autocategorización (que puede considerarse un desarrollo de la teoría de la identidad social, véase Massenzana, 2017, Scandroglio et al., 2008) las personas nos autocategorizamos como miembros de un grupo, y cuando la pertenencia a un grupo adquiere relevancia para la identidad, se activa un determinado tipo de comportamiento: nos comportamos así porque pensamos que así se han de comportar los miembros del grupo al que pertenecemos. El enlace entre el comportamiento más «personal» (o psicológico) y el comportamiento más determinado por el grupo (o sociológico) se da en el autoconcepto. De acuerdo con Massenzana (2017: 40) el autoconcepto podría definirse como la percepción que la persona tiene de sí misma, de las relaciones que tiene con otras y del valor de las propias características. Siguiendo los postulados de la psicología humanista de C. Rogers, las personas actuamos buscando reforzar nuestra autoestima a partir de lo que dicho autor denomina «refuerzos positivos». Por lo tanto, tenderemos a desarrollar aquellos comportamientos que nuestro grupo de referencia valore positivamente. En términos de Ross (2013), construimos nuestra autoestima realizando «hazañas» y evitando las «antihazañas». Según Massenzana (2017: 46):

Las hazañas son aquellas circunstancias, méritos, y virtudes que le dan la oportunidad a la persona de sentirse orgullosa de sí misma, y que le proporcionan prestigio social. Mientras que las anti-hazañas son aquellas otras situaciones que provocan que el individuo se avergüence, se auto-desprecie, se sienta menos valioso, y que le quitan también su prestigio social.

¿Qué puede considerarse una hazaña y una antihazaña? ¿Existen comportamientos que puedan considerarse que son universalmente aceptados en todas las culturas, y, por lo tanto, para todos los grupos de referencia? En los últimos ańos han tenido un importante desarrollo las disciplinas de la psicología y la antropología evolutiva ${ }^{20}$. De forma muy sucinta, podría decirse que estas arrojan luz sobre nuestro comportamiento, individual y colectivo, remitiéndose a nuestro pasado colectivo de primates. Aquellos rasgos y comportamientos que han sobrevivido lo han hecho porque, en algún momento, resultaban útiles para la adaptación al medio, que no era desde luego estable sino cambiante ya que el clima, la vegetación y las especies animales con las que han interactuado los seres humanos han ido cambiando en función de los cambios climáticos y las migraciones. Al fin y al cabo, la teoría más aceptada en la actualidad parte de la idea de que los seres humanos son un tipo de primate (Homo sapiens) que surgió en un tipo de hábitat muy determinado (este de África) pero que por su capacidad de adaptación acabó colonizando todo el planeta Tierra. Pues bien, desde esta perspectiva el que los seres humanos seamos sociales nos hace, al igual que otros seres vivos, especialmente sensibles a las jerarquías. Podría

${ }^{20}$ Sobre psicología evolutiva puede verse una introducción en Neetle, 2009; respecto a la antropología evolutiva, la conocida obra de Harari (2016) puede ser una introducción al área. 
decirse, de alguna manera, que «la estructura social no es una construcción social $»^{21}$. Como animales sociales los humanos necesitamos al grupo para poder sobrevivir. $Y$ de nuestra posición en la jerarquía del grupo depende, en gran medida, el acceso que tenemos a los recursos y, en último término, a una mejor o peor calidad de vida. $\mathrm{Si}$ en biología se habla, por ejemplo, de machos o hembras alfa, plantear, como hace la sociología, que de nuestra posición en la estructura social dependen nuestras condiciones vitales no es muy distinto. Los seres humanos somos seres preocupados por las jerarquías, y no sólo porque nos guste leer la prensa del corazón o repasar las clasificaciones de las carreras, sino porque de nuestra reputación en el grupo en el que vivimos dependen nuestras oportunidades vitales. Desde la perspectiva de la psicología y la antropología evolutiva, era muy preocupante ser percibidos como personas no dignas de fiar, pues si el grupo nos condenaba al ostracismo teníamos pocas probabilidades de seguir con vida fuera del grupo. En todas las culturas existe un patrón común, un «universal cultural» que hace que unos individuos sean vistos como buenos miembros de una comunidad, y es lo que se podría denominar comportamiento altruista (selfless, véase Storr, 2018). Así, podría plantearse que «toda sociedad o grupo humano valora positivamente a quienes son capaces de hacer sacrificios por el grupo, y valora negativamente a quienes no se muestran dispuestos a hacerlos». El altruismo nunca es genérico, sino que está determinado por el grupo de pertenencia, y de acuerdo con los últimos desarrollos en el estudio del cerebro humano (Eagleman, 2017) basta con excluir o incluir a un determinado colectivo en la definición colectiva del «nosotros» para que cambie el comportamiento de un grupo hacia él. Por lo tanto, y es lo que desarrollaremos a continuación, uno de los motivos por los que se explica el crecimiento de las pruebas deportivas de fondo es porque ayuda a construir el sentimiento de pertenencia a un colectivo que se considera socialmente valorado ${ }^{22}$.

\section{VALORACIÓN SOCIAL DE LA IDENTIDAD Y DEPORTES DE FONDO}

La teoría de la identidad social nos ayuda a entender que, dado que los seres humanos construimos nuestra identidad de manera social, haciendo aquello que nuestro grupo considera hazañas, y evitando aquello que se considera antihazañas, en la medida en que la cultura a la que pertenecemos considera hazañas unas u otras cosas nuestra propia identidad depende del grupo en el que nos socializamos. En la medida en que cambia lo que se considera una hazaña no sólo cambia nuestra identidad, sino que cambia también aquello a lo que nos dedicamos, tanto en

${ }^{21}$ El argumento, tal y como aquí se ha expuesto, se ha extraído de Storr, 2018; la fundamentación académica del mismo puede encontrarse, por ejemplo, en Wrangham y Preston, 1996 o De Waal, 2007.

22 Superhombres y supermujeres capaces de enfrentar retos como terminar un maratón o ironman. 
nuestro trabajo como, sobre todo para lo que aquí nos ocupa, en nuestro tiempo de ocio. Lo que aquí proponemos, como hipótesis de trabajo, es que la participación en pruebas deportivas de fondo ayuda a construir una identidad social en positivo por dos motivos. En primer lugar, por la mejora de la posición personal en la escala global de valoración de los individuos; en segundo lugar, porque ello permite a la persona situarse en una situación de preeminencia en al menos una jerarquía social.

Empezando por el segundo de los motivos señalados, podría decirse que la participación en pruebas deportivas de fondo permite a quienes las terminan considerarse a sí mismos, y ser percibidos socialmente, como "triunfadores» en al menos una arena social. Desde este punto de vista, el crecimiento de la participación en pruebas deportivas de fondo se explica porque permite a un amplio número de personas poder reclamar para sí, y que la sociedad se lo reconozca como tal, la etiqueta de «triunfador/a», es decir, un lugar de preeminencia en al menos una de las distintas jerarquías sociales de las que se compone toda sociedad. En una sociedad altamente competitiva, que hace que todos tengamos que luchar por nuestro puesto, $y$ que a menudo nos sintamos "fracasados", una actividad que permita a quienes se implican en ella construir su propia identidad en términos de "triunfadores» experimentará sin duda un importante auge. En este sentido, podría decirse que, por la manera en que son realizadas y percibidas estas pruebas en la actualidad, resultan más inclusivas que muchas otras modalidades deportivas ${ }^{23}$. Lo que explicaría, desde este punto de vista, el auge de la participación popular en pruebas deportivas de fondo es que estas se han reconvertido en los últimos cuarenta ańos: inicialmente eran una actividad de ocio que sólo permitía construir su identidad social en positivo a unas pocas personas, aquellas que, por diversos motivos, tenían facilidad para triunfar en las mismas ${ }^{24}$. Así, a quien se le daba bien bailar en su tiempo libre bailaba; quien era bueno en la música tocaba en una agrupación musical; quien era bueno jugando al tenis jugaba al tenis; mientras que quien era bueno corriendo se dedicaba a correr. En la actualidad, y dicho sea de una manera cercana al lenguaje coloquial, hay personas que se dedican a actividades como el triatlón o correr maratones no porque sean especialmente buenas en ello, sino porque basta con ser capaz de terminar estos eventos para ser percibido socialmente como «campeón/a», mien-

${ }^{23}$ Así, por ejemplo, en los deportes en que dos equipos se enfrentan en un partido, la mitad de los participantes en una competición serán «los perdedores». Por el contrario, las pruebas de fondo tienden a transmitir la idea de que «todo el que termina es un triunfador/a, o un campeón/a». En el que es posiblemente el evento de estas características más multitudinario, el maratón de Nueva York, participan más de 50000 personas. Obviamente, sólo una es la ganadora absoluta; si empezamos a desglosar en distintas categorías de grupos de edad, masculinos y femeninos, y si incorporamos a los tres primeros, el número podría ampliarse, pero es en cualquier medida ridículo en comparación con el total de participantes. Sin embargo, buena parte de los participantes se sienten como «triunfadores», y reciben una medalla por su participación, que lucen con orgullo y por la que reciben algún tipo de recompensa simbólica: desde acceso gratuito al transporte público a felicitaciones espontáneas de los transeúntes por haber logrado terminar.

${ }^{24}$ Esta vendría a ser la distinción que se hace en algunos entornos frecuentados por corredores entre atletismo y running. 
tras que, por ejemplo, hace falta ser bastante bueno jugando al fútbol para que se te perciba como "campeón/a» en ese terreno. Obviamente, la innovación social, que en el terreno del deporte se traduce en la aparición de nuevos deportes y modalidades deportivas, también influye en este fenómeno. El que se multipliquen las modalidades deportivas no sólo multiplica las posibilidades de destacar, sino que, especialmente en las fases de consolidación, permite «triunfar en una actividad incluso a quien tiene pocas capacidades para ella ${ }^{25}$.

Por otro lado, en la medida en que existe una homología entre el ethos de las pruebas deportivas de fondo y el de las sociedades contemporáneas, otro motivo que ayuda a explicar el auge de las pruebas deportivas de fondo es que la finalización de las mismas convierte a quienes lo logran en miembros de un grupo que es positivamente valorado por la sociedad. Es decir, no es sólo que mejore tu valoración social porque puedas ser un "buen corredor/triatleta/trail-runner», sino que ya el simple hecho de participar en estas disciplinas, en la medida en que muestra tu compromiso con algunos de los valores en los que se basan nuestras sociedades, te convierte en un buen miembro de la sociedad. El deporte en general es una actividad socialmente valorada, porque se asocia a la salud, a la disciplina, a la obtención de un cuerpo acorde a los cánones estéticos imperantes. Pero, además, se ha generalizado la idea de que las personas que practican deportes de fondo tienen el tipo de actitud y de valores adecuados para para los tiempos modernos. La subcultura propia de muchos deportes de fondo se centra en que, mediante el entrenamiento y el cuidado de la alimentación, es posible convertirse en «la mejor versión de ti mismo ${ }^{26}$ ", que coindice con la idea del "perfectible self», la idea de la existencia de un "yo" que puede mejorarse mediante la lucha y el sacrificio constante, que Storr (2018) relaciona en sus orígenes con los griegos pero que en la actualidad se manifiesta en la idea del perfeccionismo. La participación en pruebas deportivas de fondo permite construir una narrativa que nos convierte en los héroes de una epopeya griega, que mediante esfuerzo, constancia y sacrificio triunfa frente a las adversidades y acaban imponiendo su voluntad y alcanzando sus objetivos.

Por supuesto, algunos de los elementos que acabamos de señalar están presentes también tanto en otros deportes (las gestas en los deportes colectivos suelen

25 En términos coloquiales: si en tu barrio el único deporte que importa es jugar al fútbol, sólo habrá alguien que pueda reclamar para sí ser, por ejemplo, "el mejor en deporte del barrio». Si cuentan el fútbol, el baloncesto, el running y el triatlón, cuatro personas podrán reclamar ser «los mejores del barrio en deportes», cada una en su especialidad. En la misma lógica, cuando un deporte es muy poco popular en un entorno, y por ejemplo sólo tres mujeres de 40 a 45 años compiten en un medio maratón, no hace falta ser buena corriendo para lograr un puesto en el pódium. Por el contrario, si hay 500 participantes, seguramente será necesario tener un muy buen nivel para lograr un puesto entre las tres primeras.

${ }^{26}$ A título de mero ejemplo, portada de la Revista Runner's World España, septiembre de 2019: “Cómo ser más veloz / 4 Recetas para tener más energía / Respira mejor, sé más rápido / Siente el flow, siéntete invencible». Como se puede ver, la alusión a esta idea de "sé la mejor versión de ti mismo» está muy presente; la portada puede encontrarse en https://www.flipax.net/t7396-runner-s-world-espana-septiembre-2019-revista-pdf-gratis-atletismo. 
narrarse en esos términos) como en otras actividades de ocio. Pero si las actividades que aquí nos ocupan han experimentado un auge especialmente significativo es porque encarnan, en mayor medida que otras, estos valores. Y quizá el principal valor que encarnan estas actividades es el de la meritocracia. Las sociedades contemporáneas tienden a creer fervientemente en la idea de que el éxito es el resultado del talento y, sobre todo, del esfuerzo. Nos gusta pensar que para convertirse en millonario lo que importa es trabajar mucho y desearlo verdaderamente, y que otros factores, como la herencia o el azar, apenas tienen importancia. Los deportes de fondo encarnan estos ideales especialmente bien, mucho mejor que otro tipo de deportes, por diversos motivos. En primer lugar, porque el carácter masivo con el que se realizan actualmente elimina barreras de acceso (el azar no cuenta y si verdaderamente eres bueno acabarás destacando ${ }^{27}$ ). No puedes medirte a las estrellas de un deporte en disciplinas como el fútbol o el baloncesto, por supuesto no puedes practicar tu deporte en el mismo escenario que ellos. Sin embargo, en maratones y triatlones podrías, teóricamente, competir contra ellos, y en muchos eventos ciclistas puedes comparar tu rendimiento con el de las estrellas del deporte ${ }^{28}$. Además, especialmente para los no iniciados, los deportes de fondo parecen ser un ámbito en que cuenta mucho más el esfuerzo y el trabajo (el entrenamiento) que la herencia (la dotación genética). Y aunque en realidad no sea asi ${ }^{29}$, cuando los retos se conciben en términos de "terminar» una prueba podría decirse, por ejemplo, que terminar un ironman o un maratón no es que esté al alcance tan sólo de unos pocos superdotados genéticamente, sino que muchas personas, si son capaces de someterse a la disciplina y el esfuerzo de un plan de entrenamiento adecuado, podrán terminar la prueba, en su propio tiempo. "Capacidad de sacrificio, esfuerzo, disciplina», por un lado, y "personalización de las metas y logros a alcanzar, autoconocimiento de los propios límites, de cuándo forzar y cuándo levantar el pie», por otro, podría decirse que el ethos de las pruebas deportivas de fondo reúne dos de los conjuntos de valores más queridos en nuestra sociedad. Por ello, en la medida en que en las sociedades contemporáneas la participación en este tipo de eventos permite construir la identidad social en positivo, ello explica el crecimiento de este tipo de eventos.

${ }^{27}$ Por el contrario, se plantea que en otros deportes para destacar necesitas que se te dé una oportunidad, y que eso depende, en muchos casos, de las conexiones, lo que podría considerarse algo relacionado con el azar o con la herencia.

${ }^{28}$ La Etapa del Tour es una prueba ciclo deportiva para aficionados que se realiza cada ańo en el mismo recorrido, y unos días antes o después, que la prueba para profesionales. Aplicaciones para GPS como Strava permiten comparar al ciclista aficionado sus tiempos, por ejemplo en una subida famosa, con los que realizan las estrellas del ciclismo.

${ }_{29}$ Por poner un ejemplo, para correr un maratón en menos de dos horas y 10 minutos (en la actualidad, en Espańa, hay que obtener un tiempo cercano a ese para clasificarse para unos Juegos Olímpicos) no basta con entrenar mucho, cuidarse y hacer régimen: hacen falta unas condiciones genéticas que muy pocos tienen. 


\section{CONCLUSIONES}

El objetivo de este trabajo era el de plantear cómo podía abordarse, desde la sociología, el fenómeno de la creciente participación popular en pruebas deportivas de fondo: ¿por qué de repente tanta gente hace tantas locuras? No existe mucha literatura sobre el tema realizada desde un enfoque propiamente sociológico, y la que existe, producto de la creciente especialización del trabajo académico, en su mayoría se limita a reproducir unos sistemas de trabajo propios de otras disciplinas, fundamentalmente en su versión anglosajona. Como ya hemos señalado, la literatura existente ayuda a comprender, por ejemplo, los factores que determinan el rendimiento deportivo, por qué unas personas se orientan hacia unas actividades de ocio, o hacia unos deportes, y otras hacia otras, o hacia otros deportes. Pero la falta de una visión ampliamente comparativa, tanto histórica como geográficamente, limita las posibilidades de este tipo de literatura para entender la cuestión, específicamente sociológica, de por qué unas prácticas sociales cobran auge y otras decaen.

El marco teórico que domina el análisis de los fenómenos de implicación intensa en actividades de ocio es la perspectiva del ocio serio. La idea de que las personas «invierten» más en aquellas actividades que les permiten construir una identidad social en positivo es potente, y ayuda a entender muchos fenómenos, pero sería necesaria una mayor interrelación entre esta perspectiva y otras perspectivas desarrolladas desde el análisis sociológico del ocio. La idea de que el consumo, y aquello que hacemos en nuestro tiempo libre, que implica en la actualidad casi siempre alguna forma de consumo, configura quiénes somos se ha acabado convirtiendo en casi un lugar común, hasta el punto de que se ha convertido en parte del lema de muchas campañas de comunicación y marketing ${ }^{30}$. Al fin y al cabo, podría plantearse que esta idea de que «las personas invierten más en aquellas actividades de ocio que les permiten construir una identidad social en positivo" no es más que una variante de un fenómeno más amplio: a la hora de elegir entre un champú u otro, entre un destino turístico u otro, entre dedicar nuestro tiempo de ocio a una $\mathrm{u}$ otra actividad, optamos por aquellas actividades que nos permiten construir una identidad social más positiva. Aunque no es ese el sentido que sus autores les dan, se trata de una perspectiva que podría considerarse que plantea que los sujetos, a la hora de elegir entre dos posibles cursos de acción, eligen aquel que maximiza su función de utilidad, con la matización de que la identidad forma parte del cálculo de la función de utilidad, con lo que recuerda a la perspectiva de la «identity economics» de Akerlof y Kranton (2010). En cualquier caso, en la medida que este tipo de perspectiva no tiene en cuenta la cuestión del poder, podría beneficiarse de lo que podría llamarse una "economía política de las identidades", que al estilo de Hernández (2017) y Davies (2015) incluya elementos de desigualdad estructural de

30 Así, por ejemplo, en Radio 3, de Radio Nacional de España, se puede escuchar «eres lo que escuchas», y en otros ámbitos se dice «somos lo que comemos». 
poder en la configuración de las identidades y, por tanto, en último término, en la elección de prácticas de ocio.

En este mismo sentido, y quizá es una de los mayores déficits de la perspectiva del ocio serio, es necesario enmarcar las prácticas de ocio en el continuo difuso de prácticas de ocio y trabajo, a los que se alude a veces con conceptos como los de prosumo (producción y consumo), gamificación y/o gig economy ${ }^{31}$, y entender que las fronteras entre "ocio serio" (serious leisure) y trabajo pasional (devotee work) son porosas, y que la economía política actual fomenta esta confusión, pues de alguna manera permite no sólo trabajadores más implicados, sino también más baratos. Y es que, sin ir más lejos, la propia actividad académica puede entenderse, en muchos casos, como la implicación intensa en actividades que no tienen una recompensa clara en términos monetarios, pero que se hacen porque se encuentra otro tipo de satisfacciones, por lo que la diferenciación entre actividades de ocio y de trabajo se hace en ocasiones difícil. En la medida en que son cuestiones relacionadas con el poder las que hacen que una misma actividad, que se realiza en muchos casos de manera pasional, se considere ocio en unos casos y trabajo en otros ${ }^{32}$, creemos que es necesario incorporar la perspectiva estructural para el estudio de estas cuestiones. En definitiva, lo que estamos planteando implica remarcar la idea de que es necesario ir superando la que es quizá la división más enraizada en la división del trabajo en las Ciencias Sociales en los últimos 50 años, la que diferencia entre fenómenos económicos, objeto de estudio de las Ciencias Económicas, y el resto, que serían objeto de estudio para, entre otras, la Sociología o la Antropología. No es tan sólo que los fenómenos «del ocio» (no económicos) puedan incluir también aspectos «de negocio» (económicos). Es que a menudo es muy difícil separar lo uno de lo otro.

Lo que hemos pretendido ofrecer en este artículo han sido tan sólo unos elementos para reflexionar sobre el ocio. Aunque se ha titulado «elementos para un análisis sociológico", lo cierto es que al intentar encontrar respuestas a las preguntas que nos planteábamos ha sido necesario recurrir a un amplio abanico de disciplinas, desde la economía política a la psicología, la neurociencia o la fisiología del deporte. La realidad es la que es, multifacética, y las disciplinas no son más que perspectivas para acercarse al conocimiento de la misma. En las últimas décadas se ha generalizado una manera de hacer Ciencia Social que, siguiendo el modelo de las Ciencias Naturales, se basa en la extrema especialización y en la aplicación de un modelo replicable basado en muestras estadísticas. Quizá una de las potencialidades del estudio del deporte, en cuanto que actividad de ocio, es que ayude a superar este modelo, aunque aún no sepamos cuál es el que lo debería sustituir. Como se ha intentado exponer, si analizamos el deporte a nivel individual, el rendimiento deportivo es una disciplina cercana a las Ciencias Médicas; por el contrario, si nos movemos, por ejemplo, en el ámbito de los discursos en prensa acerca de las victo-

\footnotetext{
${ }^{31}$ Cuya traducción sería algo así como «economía del trabajito / del cáncamo».

${ }^{32} \mathrm{Y}$ resulta difícil decir si el que haya más o menos pasión influye en que sea considerada ocio o trabajo.
} 
rias y derrotas de los equipos nacionales, el análisis puede hacerse desde perspectivas cercanas a los estudios tradicionales en Ciencias Sociales. Sin embargo, cuando analizamos la práctica deportiva en cuanto que fenómeno social, se abre un interesante campo para el trabajo multidisciplinar. ¿Los atletas del este de África (Kenia, Etiopía) son tan buenos en maratón por su genética o por factores culturales? La respuesta a esta pregunta, que incluye sin duda cuestiones sociales y cuestiones biológicas, podría ser una oportunidad para desarrollar una perspectiva verdaderamente interdisciplinar sobre el deporte ${ }^{33}$.

Terminaremos volviendo a la pregunta con la que comenzamos: ¿cómo se explica el auge de las pruebas deportivas de fondo? Para muchos de los participantes, que las viven como actividad de ocio serio, porque los cambios recientes en las sociedades hacen que la práctica de deportes de fondo ayude a construir la identidad social en positivo más de lo que lo haría dedicar ese tiempo y esfuerzo a otras actividades. Permiten autopresentarnos como personas dinámicas, que se esfuerzan, se sacrifican y se cuidan, entre otros motivos. Para otros participantes, se trata de actividades que podrían considerarse como "ocio basado en proyectos»: no es una actividad que defina radicalmente quienes son, pero sí que es una más en la lista de «cosas que hacer para hacer un hombre (o una mujer) de provecho». En tercer lugar, para otro grupo de participantes estas actividades se practican porque permiten la acumulación de capital simbólico y relacional que, en el futuro, podría convertirse en un recurso económico. Dicho de forma resumida: en la actualidad, parece más fácil, por ejemplo, empezar a correr maratones a los 30 ańos y acabar a los 40 viviendo de ello (montando una tienda, una empresa de entrenamiento) que no empezar otras actividades de ocio como puedan ser la música o la práctica de otros deportes (fútbol o baloncesto, los más consolidados) y acabar viviendo de ello 10 años después. Por último, el auge de estas actividades tiene que ver también con que son vistas, desde empresas e instituciones, como un ámbito en el que desarrollarse profesionalmente, ganar dinero y, en términos políticos, ganar "puntos», ya que se entiende que se fomentan hábitos de vida saludable, valores positivos y otras cuestiones. Lo que acabamos de mencionar señala tanto un marco teórico desde el que sería posible abordar el estudio sociológico de la participación popular en pruebas deportivas de fondo como algunas posibles líneas de investigación desde la Sociología, identificando, por ejemplo, tipologías de participantes. Esperamos con ello haber contri-

33 Si intentamos dar una respuesta desde la perspectiva que aquí hemos planteado, diríamos en primer lugar que si tantos keniatas (o etíopes) practican maratón ello se podría explicar porque correr en maratones les permite construir en positivo su identidad social. Ello no implicaría una separación clara entre ocio y negocio, pues quizá se empieza como hobby, pero siendo conscientes de que quizá te inviten a correr en Europa, si eres lo bastante bueno; y ello también podría considerarse "construir en positivo la identidad social». Y el éxito de estos atletas tendría que ver tanto con aspectos genéticos (tienen buenos genes para correr) como culturales: dado que el maratón es el deporte rey en esos países, los jóvenes se esfuerzan para convertirse en maratonianos como en otros países se esfuerzan por convertirse en futbolistas. 
buido humildemente a avanzar en el conocimiento de esta manifestación del fenómeno deportivo en las sociedades contemporáneas.

Recibido: 23 de mayo de 2019, aceptado: 10 de julio de 2019 


\section{REFERENCIAS}

Akerlof, G. y Kranton, R. (2010), Identity Economics. Oxford, Reino Unido: Oxford University Press.

Allen, E.J., Dechow, P.M., Pope, D.G. y Wu, G. (2016). «Reference-dependent preferences: Evidence from marathon runners». Management Science, 63(6): 1657-1672.

Barbieri, C. y Sotomayor, S. (2013). «Surf travel behavior and destination preferences: An application of the Serious Leisure Inventory and Measure». Tourism Management, 35: 111-121.

Comisión Europea (2015): El Eurobarómetro sobre el deporte revela un grado elevado de inactividad en la UE, nota de prensa, disponible en http://europa.eu/rapid/press-release_IP-14300_es.htm.

Consejo Superior de Deportes (CSD) (2015): Encuesta de hábitos deportivos en España 2015, Madrid, Ministerio de Educación, Cultura y Deporte, disponible en http://www.csd.gob.es/csd/ sociedad/encuesta-de-habitos-deportivos/encuesta-de-habitos-deportivos-en-espana-2015/.

DAvies, W. (2015): The happiness industry: how the government and big business sold us well-being. Londres: Verso.

De WaAl, F. (2007). El mono que llevamos dentro. Barcelona: Tusquets.

Eagleman, D. (2017). El cerebro. Barcelona: Anagrama.

Elkington, S. y Stebbins, R.A. (2014). The serious leisure perspective: An introduction. Routledge.

Engelhardt, M., Neumann, G., Berbalk, A. y Reuter, I. (1998). "Creatine supplementation in endurance sports». Medicine and science in sports and exercise, 30(7): 1123-1129.

FAIRER-Wessels, F.A. (2013). «Motivation and behaviour of serious leisure participants: the case of the Comrades Marathon. South African journal for research in Sport». Physical Education and Recreation, 35(2): 83-103.

Fairley, S., Gibson, H. y Lamont, M. (2018). «Temporal manifestations of nostalgia: Le Tour de France». Annals of Tourism Research, 70: 120-130.

Fernández Macías, M.Á., Godoy-Izquierdo, D., Jaenes Sánchez, J.C., Bohórquez GóMEZ-Millán, M.R. y VÉlez Toral, M. (2015). Flow y rendimiento en corredores de maratón.

García Ferrando, M. (2006). «Veinticinco años de análisis del comportamiento deportivo de la población española (1980-2005)». Revista Internacional de Sociología, 64(44): 15-38.

García García, E. (2016). «Construcción y validación de un instrumento para la medición de dimensiones sociales en las prácticas deportivas de una subcultura deportiva específica: el triatlón de larga distancia». Sociologiados. Revista de investigación social, 1(2): 113-134.

Getz, D. y McConnell, A. (2011). «Serious sport tourism and event travel careers». Journal of sport management, 25(4): 326-338.

Gould, J., Moore, D., McGuire, F. y Stebbins, R. (2008). «Development of the serious leisure inventory and measure». Journal of Leisure Research, 40(1): 47-68.

Gould, J., Moore, D., Karlin, N.J., Gaede, D.B., Walker, J. y Dotterweich, A.R. (2011). «Measuring serious leisure in chess: Model confirmation and method bias». Leisure Sciences, 33(4): 332-340.

Harari, Y.N. (2014). Sapiens. De animales a dioses: Una breve historia de la humanidad. Barcelona: Debate. 
Hernández, E. (2017). «La sociedad de la excepción», en E. Hernández, Los límites del deseo. Instrucciones de uso del capitalismo del siglo XXI. Madrid: Clave Intelectual.

Hungenberg, E. y Gould, J. (2015). "*Serious Leisure and Personality Dimensions in Club Sport Athletes» The Sport Journal, vol. 21, http://thesportjournal.org/article/serious-leisure-and-personality-dimensions-in-club-sport-athletes/.

Jaenes, J.C., Godoy, D. y Román, F.M. (2009). «Personalidad resistente en maratonianos: un estudio sobre el control, compromiso y desafío de corredoras y corredores de maratón». Revista de Psicología del Deporte, 18(2).

Kokolakakis, T., Lera-López, F. y Panagouleas, T. (2012). «Analysis of the determinants of sports participation in Spain and England». Applied Economics, 44(21): 2785-2798.

Lıu, H. (2014). «Personality, leisure satisfaction, and subjective well-being of serious leisure participants». Social Behavior and Personality: an international journal, 42(7): 1117-1125.

Liu, H.S., Caneday, L. y Tapps, T. (2013). «An exploratory study of serious leisure and lifestyle for amateur athletes». LARNet: The Cyber Journal of Applied Leisure and Recreation Research, 16(3): 13 .

MassenZana, F.B. (2017). «Autoconcepto y autoestima: ¿sinónimos o constructos complementarios?» $\{P S O C I A L\}, 3(1): 39-52$.

Nettle, D. (2009). Personality: What makes you the way you are. Oxford University Press.

Novatos del Running (2018). Calendario y datos sobre la evolución de los 32 maratones en España (en línea): http://www.novatosdelrunning.es/calendario-y-datos-sobre-la-evolucion-de-los-32-maratones-en-espana/.

Podium Runner (2017). New U.S. Marathons and Halfs Report Reveals Participation is Declining (en línea): https://www.podiumrunner.com/new-report-2016-marathon-half-participation_165419.

Papanikos, G. (2015). «The economic effects of a marathon as a sport tourism event». Athens Journal of Sports, 2(4): 225-240.

Roberts, K. y FAgan, C. (1999). «Young people and their leisure in former communist countries: four theses examined». Leisure Studies, 18(1): 1-17.

Roberts, K., Povall, S. y Tholen, J. (2005). «Farewell to the intelligentsia: political transformation and changing forms of leisure consumption in the former communist countries of Eastern Europe». Leisure studies, 24(2): 115-135.

Romero, S., Iraurgi, I. y Madariaga, A. (2017). «Valoración psicométrica de la versión espańola del SLIM (Serious Leisure Inventory and Measure) en contextos deportivos». Revista de psicología del deporte, 26(2): 63-70.

Ross, M. (2013). El mapa de la autoestima. Buenos Aires: Editorial Dunken.

Ruiz-Juan, F. y SANChO, A.Z. (2014). «Análisis de la motivación en corredores de maratón españoles». Revista Latinoamericana de psicología, 46(1): 1-11.

Scandroglio, B., Martínez, J.S.L. y Sebastián, M.C.S.J. (2008). «La Teoría de la Identidad Social: una síntesis crítica de sus fundamentos, evidencias y controversias». Psicothema, 20(1): 80-89.

Shipway, R., King, K., Lee, I.S. y Brown, G. (2016). «Understanding cycle tourism experiences at the Tour Down Under». Journal of sport \& Tourism, 20(1): 21-39.

Shipway, R. y Holloway, I. (2010). «Running free: Embracing a healthy lifestyle through distance running». Perspectives in public health, 130(6): 270-276. 
Shipway, R. y Jones, I. (2007). «Running away from home: Understanding visitor experiences and behaviour at sport tourism events». International Journal of Tourism Research, 9(5): 373-383.

Shipway, R. y Jones, I. (2008). «The great suburban Everest: An 'insiders' perspective on experiences at the 2007 Flora London Marathon». Journal of Sport \& Tourism, 13(1): 61-77.

Sleivert, G.G. y Row Lands, D.S. (1996). «Physical and physiological factors associated with success in the triathlon». Sports Medicine, 22(1): 8-18.

SOTOMAYOR, S. y BARbieri, C. (2016). «An exploratory examination of serious surfers: Implications for the surf tourism industry». International Journal of Tourism Research, 18(1): 62-73.

Stebbins, R.A. (1976). «Music among friends: The social networks of amateur musicians». International review of Sociology, 12 (series II): 52-73.

Stebbins, R.A. (1977). «The amateur: Two sociological definitions». Pacific Sociological Review, 20(4): 582-606.

Stebbins, R.A. (1982). «Serious Leisure: A Conceptual Statement». The Pacific Sociological Review, 25 (2): 251-272.

Stebbins, R.A. (2005). «Project-based leisure: Theoretical neglect of a common use of free time». Leisure Studies, 24: 1-11.

Stebbins, R.A. (2006). Serious leisure. In A handbook of leisure studies (pp. 448-456). London: Palgrave Macmillan.

Stets, J.E. y Burke, P.J. (2000). «Identity theory and social identity theory». Social psychology quarterly: 224-237.

Storr, W. (2018). Selfie: How the West became self-obsessed and what it's doing to us. Nueva York: The overlook press.

Wrangham, R.W. y Peterson, D. (1996). Demonic males: Apes and the origins of human violence. Houghton Mifflin Harcourt.

Zahumensky, M. (2018). Almost half million people finished Ironman race (en línea): https:// instarea.life/so-how-many-ironmans-there-is-8223alde33d5. 